\title{
Age-related changes in LTP and antioxidant defenses are reversed by an $\alpha$-lipoic acid-enriched diet
}

\author{
Bernadette M. McGahon ${ }^{\mathrm{a}}$, Donen S.D. Martin ${ }^{\mathrm{a}}$, David F. Horrobin ${ }^{\mathrm{b}}$, Marina A. Lynch ${ }^{\mathrm{a}, *}$ \\ ${ }^{a}$ Department of Physiology, Trinity College, Dublin, Ireland \\ ${ }^{\mathrm{b}}$ Laxdale Research Ltd., Stirling, Scotland
}

Received 2 June 1999; received in revised form 4 August 1999; accepted 4 August 1999

\begin{abstract}
Among the age-related changes identified in rat hippocampus are impairments in LTP and glutamate release. These deficits have been coupled with decreased arachidonic acid concentration. In this study we compared LTP and glutamate release in groups of aged and young rats fed for 8 weeks on a control diet or on a diet enriched in $\alpha$-lipoic acid. Dietary supplementation in aged rats restored hippocampal arachidonic acid concentration to levels observed in tissue prepared from young rats. We observed that aged rats that received the experimental diet sustained LTP in perforant path-granule cell synapses in a manner indistinguishable from young rats whereas the age-related impairment in glutamate release was reversed in synaptosomes prepared from dentate gyrus obtained from these rats. The evidence presented supports the hypothesis that the $\alpha$-lipoic acid-enriched diet has antioxidant properties, because the age-related increase in superoxide dismutase activity and decrease in $\alpha$-tocopherol concentration were reversed. The finding that the age-related increase in interleukin-1 (IL-1) $\beta$ concentration was also reversed suggests a possible role for this cytokine in ageing. (C) 2000 Elsevier Science Inc. All rights reserved.
\end{abstract}

Keywords: Long-term potentiation; $\alpha$-Lipoic acid; Arachidonic acid; Glutamate release; Hippocampus; IL-1

\section{Introduction}

It is has been shown repeatedly that maintenance of long-term potentiation (LTP) is impaired in aged rats $[1,2$, $12,13,18,21,25,28,29]$. In contrast to this agreement the effects of age on induction of LTP remain unclear, with evidence of a deficit reported by some groups $[21,25,29]$ but not others [1,14].

Among the age-related changes that correlate with the impairment in LTP in dentate gyrus is a decrease in arachidonic acid concentration $[21,25,28,29]$, a proposed retrograde messenger in LTP $[11,19]$. Because arachidonic acid contributes to membrane fluidity, a decrease in its concentration is likely to be accompanied by a decrease in membrane fluidity, and this, in turn, is likely to impact on several membrane-associated functions that play a role in expression of LTP [19]. One of these membrane-associated functions is transmitter release, an increase of which has been

\footnotetext{
* Corresponding author. Tel.: +1-353-1-608-1770; fax: +1-353-1679-3545.

E-mail address: lynchma@tcd.ie (M.A. Lynch)
}

coupled with maintenance of LTP in perforant path synapses $[6,8,24,25]$. Evidence from this laboratory has consistently shown that there is an age-related decrease in $\mathrm{KCl}$-stimulated release of glutamate in synaptosomes prepared from dentate gyrus, whereas an attenuated release response to $\mathrm{KCl}$ has been observed in synaptosomes prepared from aged rats in which LTP was compromised [21, 25]. It is significant that the impairment in LTP and the compromise in transmitter release were reversed when arachidonic acid concentration was restored in aged rats fed on a diet supplemented with arachidonic acid and $\gamma$-linolenic acid [25]. It is therefore a major objective to establish the underlying cause of the age-related decrease in arachidonic acid concentration.

Recent evidence is consistent with the idea that increased lipid peroxidation is primarily responsible for the decrease in arachidonic acid concentration and that this is likely to be triggered by the proinflammatory cytokine, IL- $1 \beta$ or increased production of reactive oxygen species [28,29]. These proposals are consistent with the findings that (a) these age-related changes are reversed by dietary supplementation with the antioxidant vitamins $\mathrm{E}$ and $\mathrm{C}$ [28] and 
(b) that a number of age-related changes in neuronal function have been attributed to accumulation of reactive oxygen species, arising from either increased formation of reactive oxygen species, compromised ability of the aged brain to cope with oxidative stress, or both $[10,35,40]$.

In an effort to establish a causal relationship between age-related changes in synaptic function and age-related changes in handling of oxidative processes in cells, we assessed the ability of a diet, enriched in $\alpha$-lipoic acid, to reverse age-related changes in a number of parameters. $\alpha$-Lipoic acid is a naturally-occurring free radical scavenger that has been shown to regenerate endogenous antioxidants like vitamins $\mathrm{E}$ and increase formation of glutathione [4]. Consistent with its role as an antioxidant, $\alpha$-lipoic acid reversed the oxidative stress-induced activation of $\mathrm{NF} \kappa \mathrm{B}$ [5,33] and the increased lipid peroxidation [30] that are associated with diabetic neuropathy. It has also been shown to reverse the damage induced by ischemia in CA1 pyramidal cells [9] and also the damage that accompanies reperfusion [34].

In this study, groups of aged and young rats were fed either on a control diet or on a diet enriched in $\alpha$-lipoic acid and assessed for their ability to sustain LTP. Tissue obtained from dentate gyri of these rats was analyzed for fatty acid concentration and assessed for glutamate release. We observed that dietary manipulation reversed age-related impairment in LTP and glutamate release and these changes were accompanied by restoration of fatty acid, IL- $1 \beta$ and $\alpha$-tocopherol concentrations to values observed in young rats.

\section{Experimental procedures}

\subsection{Animals}

Aged and young male Wistar rats (Bantham and Kingman, UK) were housed in groups of 2-4 under a 12-h light schedule, with the ambient temperature controlled between 22 and $23^{\circ} \mathrm{C}$. Rats were maintained in the BioResources Unit in Trinity College, Dublin, were checked daily and were subject to veterinary supervision throughout the experiment. Young and aged rats were divided into groups that were fed on 'control' or 'experimental' diet. The composition of the experimental diet was as follows: $20 \% \gamma$-linolenic-lipoic acid diol, $40 \%$ tuna oil containing $24 \%$ docosahexanoic acid and $1.7 \%$ arachidonic acid and $40 \%$ microbial oil containing $40 \%$ arachidonic acid, $3 \% \gamma$-linolenic acid and $4 \%$ dihomo- $\gamma$-linolenic acid (Laxdale Research, UK). The overall composition of the diet, in terms of polyunsaturated fatty acids, was therefore $20 \%$ of $\gamma$-linolenic-lipoic acid diol, 9.6\% of docosahexanoic acid, $16.4 \%$ of arachidonic acid, $1.2 \%$ of $\gamma$-linolenic acid and $1.6 \%$ of dihomo$\gamma$-linolenic acid. One group of aged and one group of young animals were fed for 8 weeks on $100 \mathrm{mg} / \mathrm{rat} /$ day experimental diet, referred to hereafter as ' $100 \%$ experimental diet'. A second group of young and aged animals received standard laboratory chow to which corn oil was added to ensure an isocaloric intake for all rats. A third group of aged rats received $50 \mathrm{mg} / \mathrm{rat} / \mathrm{day}$ of experimental diet (hereafter referred to as ' $50 \%$ experimental diet'). Food intake was measured for 2 weeks before the commencement of the experiment, sufficient diet was prepared for 3 or 4 days, daily allowances were stored in airtight containers at $-80^{\circ} \mathrm{C}$ and rats were offered their full daily requirement each day. At the end of the period of dietary manipulation the average ages of the aged and young rats were 22 months and 4 months respectively.

\subsection{Induction of long-term potentiation in dentate gyrus}

Rats were anesthetized with urethane; young animals initially received $1.5 \mathrm{~g} / \mathrm{kg}$ of urethane intraperitoneally (i.p.), and a further increment, if required (that did not exceed $0.6 \mathrm{~g} / \mathrm{kg}$ in any experiment) for induction of deep anesthesia, that was assessed by the absence of a pedal reflex. The mean doses ( \pm SEM) administered to young rats given the control and experimental diets were $1.92 \mathrm{~g} / \mathrm{kg}$ $( \pm 0.08)$ and $1.82 \mathrm{~g} / \mathrm{kg}( \pm 0.05)$ respectively. Aged animals received $1.2 \mathrm{~g} / \mathrm{kg}$ of urethane initially and topped up when necessary; the mean doses administered to aged rats given the control, 50\% lipoic acid and 100\% lipoic acid diets were $1.44 \mathrm{~g} / \mathrm{kg}( \pm 0.05), 1.37 \mathrm{~g} / \mathrm{kg}( \pm 0.04)$ and $1.48 \mathrm{~g} / \mathrm{kg}$ $( \pm 0.06)$, respectively.

LTP was induced in perforant path-granule cell synapses as described previously [24]. Briefly, a bipolar stimulating electrode was placed in the perforant path $(4.4 \mathrm{~mm}$ lateral to Lambda) and a unipolar recording electrode was placed in the dorsal cell body region of the dentate gyrus $(2.5 \mathrm{~mm}$ lateral and $3.9 \mathrm{~mm}$ posterior to Bregma). The depths of the stimulating and recording electrodes were adjusted to maximize the amplitude of the response; the stimulus intensity was adjusted to the value that was just sufficient to induce a population spike. Test shocks were given at $30 \mathrm{~s}$ intervals for $10 \mathrm{~min}$ before and $40 \mathrm{~min}$ after, 3 trains of stimuli (250 $\mathrm{Hz}$ for $200 \mathrm{~ms}$, with a $30 \mathrm{~s}$ intertrain interval) that was shown previously to produce saturation of LTP in perforant path-granule cell synapses.

\subsection{Tissue preparation}

At the end of the recording period, rats were killed by decapitation, the hippocampus proper and the dentate gyri were dissected, and cross-chopped slices $(350 \mu \mathrm{m} \times 350$ $\mu \mathrm{m})$ were prepared using a McIlwain tissue chopper. Samples of hippocampus and control and potentiated dentate gyri were frozen separately in $1 \mathrm{ml}$ of Krebs solution (composition of Krebs in $\mathrm{mM}: \mathrm{NaCl} 136, \mathrm{KCl} 2.54, \mathrm{KH}_{2} \mathrm{PO}_{4}$ $1.18, \mathrm{MgSO}_{4} .7 \mathrm{H}_{2} \mathrm{O} 1.18, \mathrm{NaHCO}_{3} 16$, glucose $10, \mathrm{CaCl}_{2}$ 1.13) containing $10 \%$ dimethylsulphoxide [15] and stored at $-80^{\circ} \mathrm{C}$ until required.

For analysis, slices were thawed rapidly (1.5-2 min) by 
agitation at $37^{\circ} \mathrm{C}$ and rinsed 4 times in excess fresh oxygenated Krebs solution. Fatty acid concentrations were assessed in samples of homogenate prepared from dentate gyrus of aged and young rats, whereas the crude synaptosomal pellet, $\mathrm{P}_{2}$, prepared as previously described [24] was used for analysis of glutamate release. Homogenate prepared from hippocampal tissue was used for analysis of $\alpha$-tocopherol and IL- $1 \beta$ concentrations and activity of superoxide dismutase.

\subsection{Assessment of glutamate release}

Samples of synaptosomal tissue were resuspended in ice-cold Krebs solution containing $2 \mathrm{mM}$ of $\mathrm{CaCl}_{2}$. Tissue was aliquotted onto Millipore filters $(0.45 \mu \mathrm{m})$, rinsed under vacuum and the filtrate was discarded. Tissue was incubated in $250 \mu \mathrm{l}$ of oxygenated $\mathrm{Krebs}$ solution at $37^{\circ} \mathrm{C}$ for $3 \mathrm{~min}$ and filtrate was collected and stored. Release of transmitter was then stimulated by the addition of $40 \mathrm{mM}$ of $\mathrm{KCl}$ to Krebs solution. In some cases, arachidonic acid $(1 \mu \mathrm{M})$ and ACPD $(50 \mu \mathrm{M})$ were added to the incubation medium to assess the effect of these agents on KCl-stimulated release.

Glutamate was analyzed as described [26,32]. Briefly, 96-well plates were coated with glutaraldehyde $(0.5 \%$ in $100 \mathrm{mM}$ of $\mathrm{NaH}_{2} \mathrm{PO}_{4}$ buffer, $\mathrm{pH} 4.5 ; 320 \mu \mathrm{l}$ ) by incubation for $60 \mathrm{~min}$ at $37^{\circ} \mathrm{C}$. Plates were then washed with $100 \mathrm{mM}$ of $\mathrm{NaH}_{2} \mathrm{PO}_{4}$ buffer. Triplicate samples $(50 \mu \mathrm{l})$ or glutamate standards $(50 \mu \mathrm{l} ; 50 \mathrm{nM}$ to $10 \mu \mathrm{M}$ prepared in $100 \mathrm{mM}$ of $\mathrm{Na}_{2} \mathrm{HPO}_{4}$ buffer, $\mathrm{pH}$ 8.0) were added, plates were incubated for $2 \mathrm{~h}$ at $37^{\circ} \mathrm{C}$ and washed with $100 \mathrm{mM}$ of $\mathrm{NaH}_{2} \mathrm{PO}_{4}$ buffer. To bind any unreacted aldehydes, ethanolamine (320 $\mu \mathrm{l} ; 0.1 \mathrm{M}$ in $100 \mathrm{mM}$ of $\mathrm{Na}_{2} \mathrm{HPO}_{4}$ buffer) was added to each well and incubated for $60 \mathrm{~min}$ at $37^{\circ} \mathrm{C}$. Plates were washed with phosphate-buffered saline (PBS) containing Tween-20 (0.2\% Tween; PBS-T). To block non-specific binding, samples were incubated with donkey serum for 60 $\min (200 \mu \mathrm{l} ; 3 \%$ in PBS-T). Antiglutamate antibody (raised in rabbit; $100 \mu \mathrm{l}$; 1:5,000 in PBS-T; Sigma, UK) was added to each plate, incubated overnight at $4^{\circ} \mathrm{C}$ and washed with PBS-T. Anti-rabbit horseradish peroxidase (HRP)-linked secondary antibody (95 $\mu \mathrm{l} ; 1: 10,000$ in PBS-T; Amersham, UK) was added, incubated for $60 \mathrm{~min}$ at room temperature and plates were again washed with PBS-T. 3,3',5,5'-Tetramethylbenzidine $(100 \mu \mathrm{l})$ liquid substrate was added as chromogen and incubation continued for $60 \mathrm{~min}$ at room temperature. $\mathrm{H}_{2} \mathrm{SO}_{4}(4 \mathrm{M} ; 50 \mu \mathrm{l})$ was used to stop the reaction and optical densities were determined at $450 \mathrm{~nm}$ using a multiwell plate reader. Values were calculated with reference to the standard curve, corrected for protein [7] and expressed as nmol glutamate/mg protein.

\subsection{Analysis of fatty acid concentration}

Thawed slices prepared from dentate gyrus were rinsed 3 times and then homogenized in $200 \mu$ lof fresh Krebs solution. Fatty acids were extracted into chloroform:metha- nol $(2: 1 \mathrm{v} / \mathrm{v} ; 1 \mathrm{ml})$ by vigorous shaking for $10 \mathrm{~min}$ followed by centrifugation at $1000 \times g$ for 5 min to separate the phases. The aqueous layer was discarded and the chloroform phase was evaporated under nitrogen and resuspended in ethanol for analysis. Fatty acid concentrations were analyzed as their 2-nitrophenylhydrazine (NPH) derivatives by reverse phase HPLC [22]. Derivatization was achieved by adding 2-NPH-HCl solution (0.02 M 2-nitrophenylhydrazine- $\mathrm{HCl}$ in $0.25 \mathrm{M} \mathrm{HCl}$-ethanol (1:1, v/v) and EDC solution (1-ethyl-3-(3-dimethylaminopropyl) carbodiimide hydrochloride; $0.25 \mathrm{M}$ EDC in ethanol mixed in equal volumes with $3 \%$ ethanolic pyridine), and incubated at $60^{\circ} \mathrm{C}$ for 20 min. After addition of $\mathrm{KOH}(15 \% \mathrm{w} / \mathrm{v}$ in $\mathrm{MeOH}$ : $\mathrm{H}_{2} \mathrm{O}, 80: 20$ ) samples were incubated at $60^{\circ} \mathrm{C}$ for $15 \mathrm{~min}$ and cooled in running water. Fatty acid derivatives were concentrated; n-hexane and phosphate buffer $(0.033 \mathrm{M}, \mathrm{pH} 6.4$ in $0.5 \mathrm{M} \mathrm{HCl}$ ) were added, samples were vortex mixed for $30 \mathrm{~s}$, centrifuged for $5 \mathrm{~min}$ at $1500 \times g$ and the hexane phase evaporated to dryness under nitrogen. For HPLC analysis, samples were resuspended in methanol, injected onto a Microsorb C18 column (maintained in a column oven at $30^{\circ} \mathrm{C}$ ) and fatty acids were separated in isocratic mode with a mobile phase of $85 \%$ methanol:15\% water (maintained at $\mathrm{pH} 4.5$ with $\mathrm{HCl}$ ) and detected by UV spectroscopy at $230 \mathrm{~nm}$. Aliquots of tissue were taken for protein analysis and concentrations were estimated according to standard curves and expressed as $\mathrm{nmol} / \mathrm{mg}$ protein.

\subsection{Analysis of $\alpha$-tocopherol}

$\alpha$-Tocopherol was analyzed according to the method of Vatassery [38]. Briefly, aliquots of hippocampal homogenate $(150 \mu \mathrm{l})$ were incubated in the presence of ethanol containing $0.025 \%$ butyl-hydroxytoluene $(150 \mu \mathrm{l}), 25 \%$ ascorbic acid $(70 \mu \mathrm{l})$ and $10 \%$ potassium hydroxide (135 $\mu \mathrm{l})$ for $30 \mathrm{~min}$ at $60^{\circ} \mathrm{C}$. Hexane $(540 \mu \mathrm{l})$ containing $0.025 \%$ butyl-hydroxytoluene was added, samples were vortexmixed for $1 \mathrm{~min}$ and centrifuged at $1500 \times g$ for $6 \mathrm{~min}$. The hexane phase was removed and evaporated to dryness under nitrogen; the recovery of $\alpha$-tocopherol using this procedure was between 70 and $80 \%$. For HPLC analysis, dried samples were resuspended in methanol $(150 \mu \mathrm{l})$ containing $0.025 \%$ butyl-hydroxytoluene and $30 \mu \mathrm{l}$ volumes were injected onto an Intersil C18 column. Separation of $\alpha$-tocopherol was achieved using a mobile phase of $75 \%$ acetonitrile: $25 \%$ methanol at a flow rate of $1.2 \mathrm{~mL} / \mathrm{min}$ and samples were detected by uv spectroscopy at $292 \mathrm{~nm}$. $\alpha$-Tocopherol concentration was estimated by the external standard method and expressed as $\mu \mathrm{g} / \mathrm{mg}$ tissue.

\subsection{Analysis of superoxide dismutase activity}

Superoxide dismutase activity was determined in samples of hippocampal homogenate [36]. Aliquots $(800 \mu \mathrm{l})$ of incubation buffer $(50 \mathrm{mM}$ potassium buffer $(\mathrm{pH} 7.8)$ containing $1.8 \mathrm{mM}$ xanthine, $2.24 \mathrm{mM}$ nitroblue tetrazolium 
(NBT), 40 units of catalase, $7 \mu \mathrm{l} / \mathrm{ml}$ xanthine oxidase and $1.33 \mathrm{mM}$ diethylenetriaminepentacetic acid) were added to $1.5 \mathrm{ml}$ microfuge tubes containing samples of supernatant $(100 \mu \mathrm{l})$ at different dilutions $(1: 2,1: 5,1: 10,1: 20,1: 50$ and $1: 100)$ and analyzed by uv spectroscopy at $560 \mathrm{~nm}$. Activity of superoxide dismutase was assessed as the rate of reduction of NBT, that was inhibited with increasing concentrations of protein. One unit of activity was defined as the amount of protein necessary to decrease the rate of the reduction of NBT by 50\%. Results were expressed in units of superoxide dismutase activity per mg protein.

\subsection{Analysis of IL-1 $\beta$}

IL-1 $\beta$ (IL-1 $\beta$; Genzyme Diagnostics, USA) concentrations in hippocampal homogenate were analyzed by ELISA as previously described [28]. Briefly, 96-well plates were coated with $100 \mu \mathrm{l}$ of capture antibody $(2.0 \mu \mathrm{g} / \mathrm{ml}$ of final concentration, diluted in $0.1 \mathrm{M}$ of sodium carbonate buffer, pH 9.5; monoclonal hamster anti-mouse IL-1 $\beta$ antibody), incubated overnight at $4^{\circ} \mathrm{C}$, washed with PBS containing $0.05 \%$ Tween 20 and blocked for $2 \mathrm{~h}$ at $37^{\circ} \mathrm{C}$ with $250 \mu \mathrm{l}$ of blocking buffer, (PBS, pH 7.3; $0.1 \mathrm{M}$ with $4 \%$ bovine serum albumin (BSA)). Aliquots $(50 \mu \mathrm{l})$ of samples or IL- $1 \beta$ standards $(0-1000 \mathrm{pg} / \mathrm{ml})$ were incubated for $1 \mathrm{~h}$ at $37^{\circ} \mathrm{C}$. Plates were washed and secondary antibody $(100 \mu \mathrm{l}$; final concentration $0.8 \mu \mathrm{g} / \mathrm{ml}$ in PBS containing $0.05 \%$ Tween 20 and $1 \%$ BSA; biotinylated polyclonal rabbit antimouse IL- $1 \beta$ antibody) was added and incubated for $1 \mathrm{~h}$ at $37^{\circ} \mathrm{C}$. Detection agent $(100 \mu \mathrm{l}$ of horseradish peroxidaseconjugated streptavidin; 1:1000 dilution in PBS containing $0.05 \%$ Tween 20 and $1 \%$ BSA) was added, incubated for 15 min at $37^{\circ} \mathrm{C}$. Substrate (100 $\mu$; t tetramethylbenzidine liquid substrate; Sigma, UK) was added, incubated at room temperature for $10 \mathrm{~min}$ and absorbance read at $450 \mathrm{~nm}$. Results were expressed as pg IL- $1 \beta / \mathrm{mg}$ tissue.

\subsection{Statistical analysis}

Data were analyzed, as appropriate, using either the Student's $t$-test for independent means, or by using a oneway analysis of variance (ANOVA) followed by post hoc analysis using the Newmann-Keuls test.

\section{Results}

The average food intake per rat for young rats was 22 $\mathrm{g} / \mathrm{rat} /$ day compared with $18 \mathrm{~g} / \mathrm{rat} / \mathrm{day}$ for aged rats; these values did not vary for the duration of the experiment. Mean body weights ( \pm SEM) at the end of the experimental period were $481 \mathrm{~g}( \pm 28.4)$ and $481 \mathrm{~g}( \pm 16.1)$ in the young rats fed on the control and experimental diets and $525 \mathrm{~g}$ ( \pm $15.5), 516 \mathrm{~g}( \pm 9.8)$ and $479 \mathrm{~g}( \pm 14.8)$ in the aged rats fed on the control, $50 \%$ and $100 \%$ diets respectively.

In these experiments, the stimulus strength used was that which was just sufficient to induce a spike; the mean values $( \pm$ SEM; V) were $1.53( \pm 0.31)$ and $4.69( \pm 0.19)$ in young and aged rats respectively. The mean values for EPSP slope ( $\pm \mathrm{SEM} ; \mathrm{mV} / \mathrm{ms}$ ) in young rats fed on control and experimental diets were $1.83( \pm 0.10)$ and $1.82( \pm 0.08)$ respectively. In aged rats, the values were significantly lower $(p<$ 0.05 ; student's $t$-test for independent means); the means were $1.30( \pm 0.03), 1.29( \pm 0.07)$ and $1.63( \pm 0.11)$ for the groups fed on control, $50 \%$ and $100 \%$ diets respectively. The corresponding values for the mean amplitude of the response $(\mathrm{mV})$ in young rats fed on control and experimental diets were $4.42( \pm 0.14)$ and $4.40( \pm 0.13)$. These values were higher than those recorded from aged rats, that were $3.62( \pm 0.07), 4.11( \pm 0.27)$ and $3.37( \pm 0.09)$ for rats fed on control, $50 \%$ and $100 \%$ experimental diets respectively.

Fig. 1a indicates that there was a marked increase in mean EPSP slope immediately after tetanic stimulation in young rats fed on control or experimental diet; an increase in mean EPSP slope was observed for the duration of the experiment though dietary manipulation attenuated the more persistent EPSP response (see Table 1). Fig. 1b indicates that LTP was compromised in aged rats fed on the control diet, whereas aged rats fed on either experimental diet demonstrated an ability to sustain LTP that was similar to that of young rats; we observed an age-related attenuation in both the early and later response (see Table 1).

Analysis of several fatty acids in homogenate prepared from dentate gyri indicated that there were no significant differences in concentrations between the young rats fed on control and experimental diets (Fig. 2). The data indicate that the concentration of arachidonic acid was markedly decreased in tissue prepared from aged rats fed on the control diet $(n=11)$ compared with tissue prepared from young rats $(p<0.05$; ANOVA; $n=8$ in both dietary groups). Fig. 2 shows that dietary manipulation with either the $50 \%$ diet $(n=9)$ or the $100 \%$ diet $(n=11)$ completely reversed the age-related decrease in arachidonic acid concentration. In contrast to the age- and diet-related changes in arachidonic acid, the concentration of the arachidonic acid precursor, $\gamma$-linolenic acid was unchanged. Analysis of the ratio of $\gamma$-linolenic acid to arachidonic acid indicated values of $0.15( \pm 0.03)$ and $0.20( \pm 0.06)$ in the young rats fed on control and experimental diets respectively. The corresponding values were $0.38( \pm 0.06), 0.17( \pm 0.02)$ and 0.11 $( \pm 0.02)$ in aged rats fed on the control, $50 \%$ and $100 \%$ diets respectively.

The concentration of docosahexanoic acid was markedly decreased in tissue prepared from aged rats fed on the control diet compared with tissue prepared from young rats $(p<0.05$; ANOVA). Dietary manipulation partially reversed this decrease, though concentrations were still significantly lower then those in tissue prepared from young rats. In contrast, the concentrations of linoleic and palmitic acids were significantly increased with age $(p<0.05$; ANOVA), whereas there was no significant age-, or dietrelated change in the concentration of $\alpha$-linolenic acid. The 


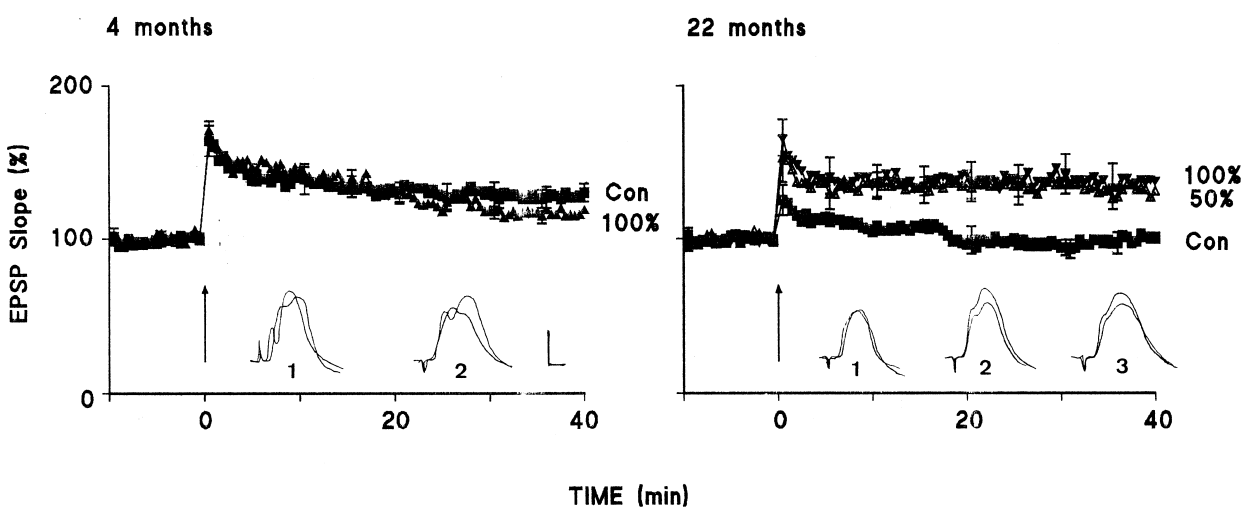

Fig. 1. The age-related impairment in LTP is restored by dietary supplementation. The mean slope of the population EPSP was markedly increased after tetanic stimulation (arrow) in both groups of young rats ( $n=8$ in each case). In contrast, there was a marked impairment in maintenance of LTP in aged rats that received the control diet $(n=11)$. Dietary manipulation with either diet $(n=9$ for the $50 \%$ diet and $n=11$ for the $100 \%$ diet $)$ reversed the age-related impairment in LTP. Mean EPSP slopes are expressed as a percentage of the values recorded in the 5 min immediately before tetanic stimulation. Standard errors are included for every tenth response and are so small as to be obscured in some cases. Sample waveforms are included for young rats (left hand panel) fed on the control (1) and experimental (2) diets, and for aged rats (right-hand panel) fed on the control (1), 50\% (2) and 100\% (3) diets; in each case responses obtained after tetanic stimulation are superimposed on responses obtained before tetanic stimulation. The scale bars represent $1 \mathrm{mV}$ and $2 \mathrm{~ms}$ refer to responses for both aged and young rats.

concentrations of palmitic and linoleic acids were also not significantly affected by diet.

Endogenous glutamate release was assessed in synaptosomes prepared from dentate gyrus in each of the experimental groups. Fig. 3a shows that unstimulated glutamate release was similar in synaptosomes prepared from untetanized and tetanized dentate gyri of both groups of young rats. Addition of $40 \mathrm{mM}$ of $\mathrm{KCl}$ to the incubation medium significantly enhanced release in untetanized tissue prepared from both groups of young rats ( $p<0.05$; ANOVA), but the enhancement was more marked in synaptosomes prepared from dentate gyrus in which LTP was sustained ( $p<$ 0.01; ANOVA). Addition of arachidonic acid and ACPD significantly increased $\mathrm{KCl}$-stimulated release of glutamate in synaptosomes prepared from untetanized tissue, but this effect was occluded in synaptosomes prepared from teta-

Table 1

Mean percentage change in epsp slope ${ }^{\mathrm{a}}$

\begin{tabular}{lcc}
\hline & $0-2$ min posttentanus & $35-40$ min posttetanus \\
\hline 4 months & & \\
$\quad$ Control diet & $157.64(6.34)$ & $127.01(2.09)$ \\
$\quad$ Experimental diet & $159.22(5.09)$ & $116.80(1.22)$ \\
22 months & & \\
Control diet & $117.76(2.99)$ & $98.88(1.04)$ \\
$50 \%$ diet & $145.68^{*}(6.10)$ & $130.26^{*}(2.07)$ \\
$100 \%$ diet & $155.33^{*}(5.88)$ & $137.04^{*}(4.66)$ \\
\hline
\end{tabular}

${ }^{a}$ Mean percentage changes in epsp slope in two time intervals posttetanus ( $0-2 \mathrm{~min}$ and $35-40 \mathrm{~min}$ ) in young and aged rats fed on control or experimental diets. The early mean percentage change in epsp slope was similar in both groups of young rats, through dietary manipulation attenuated and later response. LTP was attenuated in aged rats fet on the control diet, but this was not observed in aged rats fed on either the $50 \%$ or $100 \%$ diet. The asterisks indicate significant differences between values in rats fed on control and experimental diets $(p<0.05$; ANOVA). nized tissue (Fig. 3a and 3b). KCl-stimulated release of glutamate was markedly attenuated in synaptosomes prepared from untetanized dentate gyrus of aged rats fed on the control diet and there was no evidence of a $\mathrm{KCl}$-induced enhancement of release in synaptosomes prepared from tetanized dentate gyrus (Fig. 3c). Addition of arachidonic acid and ACPD failed to affect $\mathrm{KCl}$-induced release in either untetanized or tetanized tissue. In contrast to the lack of response in tissue prepared from aged rats fed on the control diet, $\mathrm{KCl}$ significantly increased glutamate release in synaptosomes prepared from untetanized dentate gyrus of aged rats fed on either experimental diet $(p<0.05$; ANOVA). A further enhancement of release was observed in synaptosomes prepared from tetanized tissue $(p<0.01$; ANOVA). Addition of arachidonic acid and ACPD to the incubation medium significantly increased $\mathrm{KCl}$-induced release in synaptosomes prepared from untetanized dentate gyrus $(p<0.01$; ANOVA), but this effect was occluded in synaptosomes prepared from tetanized tissue (Fig. 3d and $3 \mathrm{e})$.

Because $\alpha$-lipoic acid has been shown to have antioxidant properties and because antioxidative defenses have been shown to be compromised with age [31], we analyzed age-related changes in 3 parameters in hippocampal tissue prepared from the young and aged rats that received control or experimental diets. The data indicated that dietary manipulation in young rats exerted no effect on $\alpha$-tocopherol concentration, IL-1 $\beta$ concentration or superoxide dismutase, therefore data from the two groups of young rats are pooled in Fig. 4. We observed that the concentration of $\alpha$-tocopherol was decreased in the hippocampus of aged rats fed on the control diet, compared with young rats $(p<0.05$; ANOVA; Fig. 3a). Dietary manipulation with $100 \%$ diet 
(A) Arachidonic acid
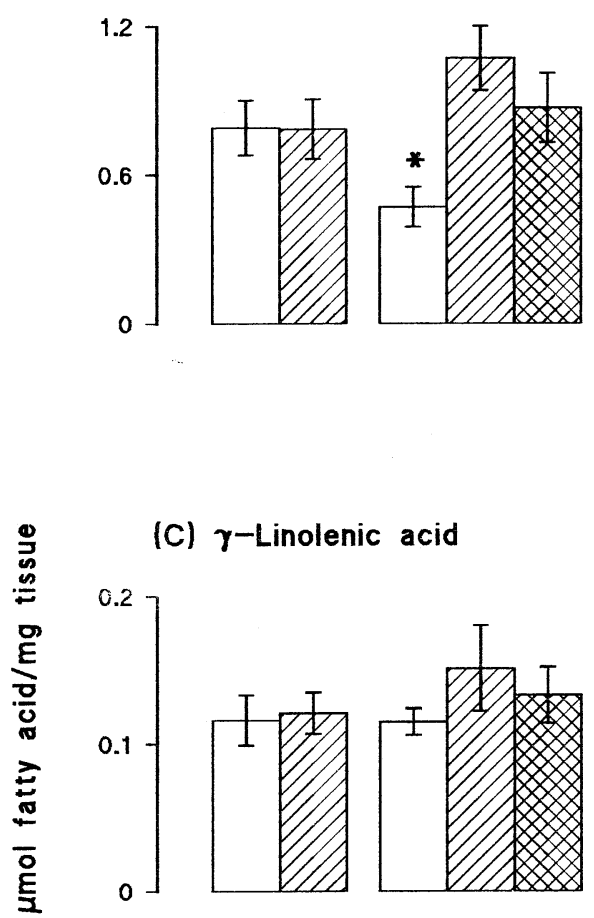

(E) Linoleic acid

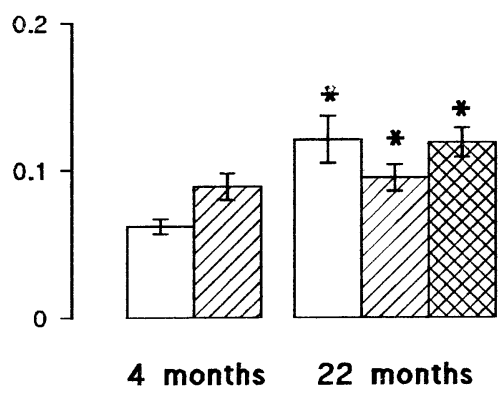

(B) Docosahexanoic acid

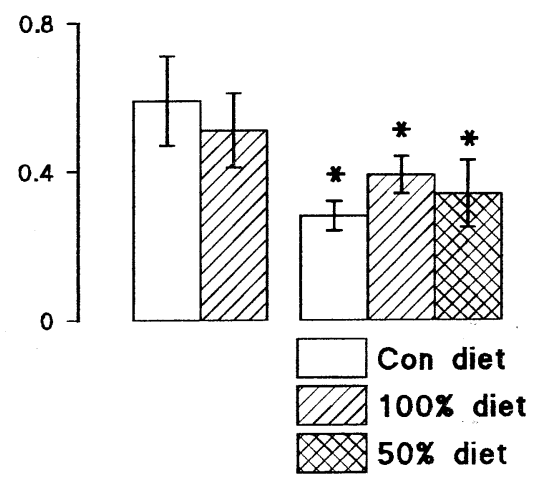

(D) Linolenic acid

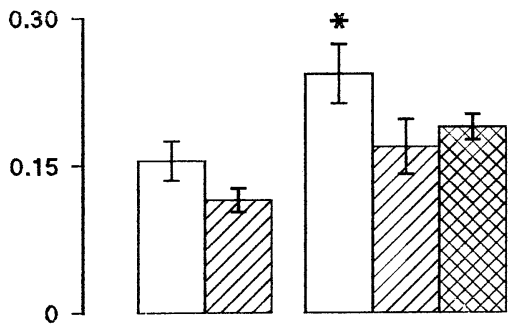

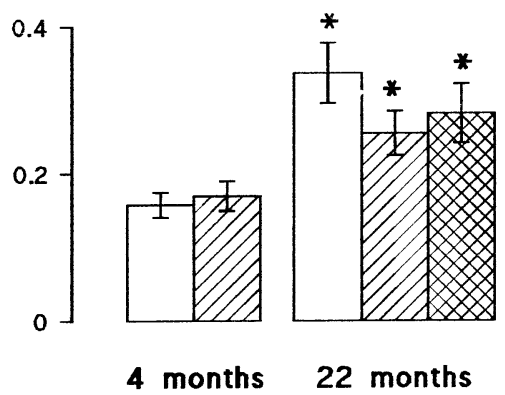

Fig. 2. Dietary manipulation reverses age-related changes in concentrations of arachidonic acid and docosahexanoic acid. Concentrations of arachidonic acid, docosahexanoic acid, linolenic acid, $\gamma$-linolenic acid, linoleic acid and palmitic acid were similar in both groups of young rats $(n=8$ in each case). The concentrations of arachidonic and docosahexanoic acids were significantly decreased in tissue prepared from dentate gyrus of aged rats that received the control diet $(n=11)$, whereas significant increases in the concentrations of linoleic acid and palmitic acid were observed $\left({ }^{*} p<0.05\right.$; Student's $t$-test for independent means). Dietary supplementation with either the 50\% $(n=9)$ or $100 \%(n=11)$ diets reversed the age-related changes in arachidonic acid and linolenic acid.

partially reversed this effect, but reversal was almost complete in tissue prepared from rats that received the $50 \%$ diet.

An age-related increase in hippocampal concentration of IL- $1 \beta$ was also observed ( $p<0.05$; ANOVA; Fig. $4 \mathrm{~b})$. We found that this effect was completely reversed by supplementation with the $100 \%$ lipoic acid diet, whereas the 50\% diet was less effective. Similarly, the activity of superoxide dismutase was significantly increased in the hippocampus of aged rats $(p<0.05$; ANOVA; Fig. 4b); this effect was completely reversed in hippocampal tissue prepared from rats that received the $100 \%$ lipoic acid diet. Enzyme activity, however, was similar in tissue prepared from aged rats that received the control diet and those that received the $50 \%$ diet.

\section{Discussion}

The objective of this study was to establish whether certain age-related changes in hippocampal synaptic func- 
(A) 4 months: Con diet

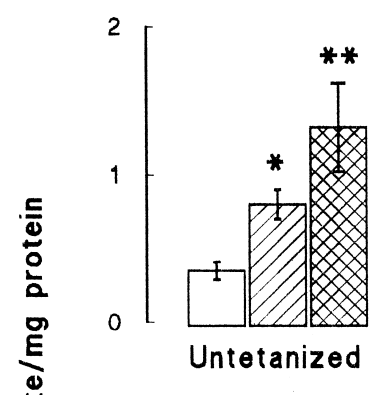

(B) 4 months: $100 \%$ diet

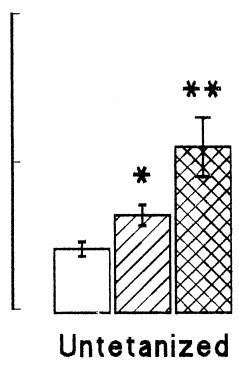

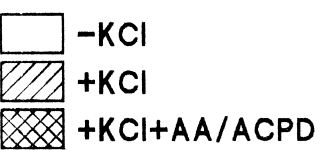

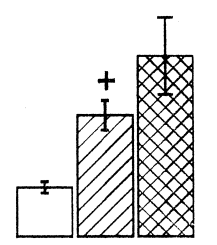

Tetanized
(C) 22 months: Con diet

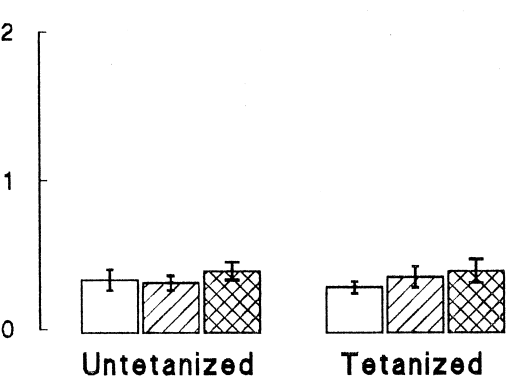

(D) 22 months: $100 \%$ diet

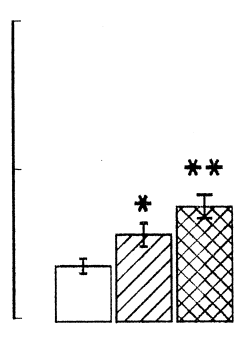

Untetanized

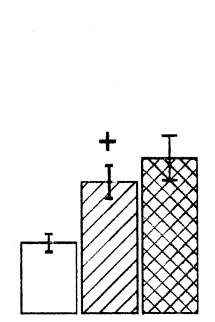

Tetanized
(E) 22 months: $50 \%$ diet

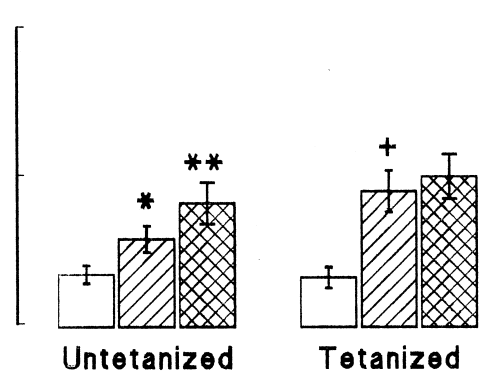

Fig. 3. Dietary manipulation reverses age-related changes in $\mathrm{KCl}$-stimulated glutamate release. $\mathrm{KCl}$ ( $40 \mathrm{mM})$ increased release to a significantly greater degree in tetanized $(* * p<0.01$; ANOVA), compared with untetanized $(* p<0.05$; ANOVA), tissue prepared from young rats that were fed either diet $(n=$ 8 in each case); arachidonic acid (AA) and ACPD significantly enhanced KCl-stimulated release in untetanized tissue $(+p<0.01$; ANOVA), but not in tetanized tissue. $\mathrm{KCl}$ failed to increased glutamate release in synaptosomes prepared from either untetanized or tetanized tissue obtained from aged rats fed on the control diet $(n=11)$. In aged rats fed on either experimental diet ( $n=9$ for $50 \%$ diet and $n=11$ for $100 \%$ diet), $\mathrm{KCl}(40 \mathrm{mM})$ increased release to a significantly greater degree in tetanized tissue (**p $<0.01$; ANOVA), compared with untetanized tissue (* $p<0.05$; ANOVA); arachidonic acid (AA) and ACPD significantly enhanced KCl-stimulated release in untetanized $(+p<0.01$; ANOVA), but not tetanized tissue.

tion might be reversed by supplementation with a diet enriched in $\alpha$-lipoic acid. The data show that dietary manipulation reversed the age-related impairment in ability of aged rats to sustain LTP in perforant path-granule cell synapses.

In this study we observed that dietary manipulation of aged rats with either experimental diet completely reversed the age-related attenuation in the initial tetanus-induced enhancement of the synaptic response. Similarly, the marked attenuation in the later response, that reflects impaired maintenance of LTP was also completely reversed by dietary manipulation.

We have previously reported that aged rats that received a diet enriched in arachidonic acid and its precursor $\gamma$-linolenic acid exhibited an ability to sustain LTP that was comparable to young rats [25]; this was accompanied by a reversal of the age-related decrease in arachidonic acid concentration. Analysis of fatty acids in dentate gyrus of the young and aged rats in the present study demonstrated that the age-related decrease in arachidonic acid concentration was also reversed by dietary manipulation with an $\alpha$-lipoic acid-enriched diet. This may be a direct effect, due to incorporation of arachidonic acid, because the diet contained arachidonic acid (16.4\%). It may also be an indirect effect because the antioxidant properties of $\alpha$-lipoic acid [4] have been shown here to be effective in hippocampal, as well as other, tissues. One reported antioxidant effect of $\alpha$-lipoic acid is its ability to regenerate vitamin E [4]; this effect of $\alpha$-lipoic acid may contribute to the observed effects, because we have reported that dietary manipulation with vitamins $\mathrm{E}$ and $\mathrm{C}$ reverse the age-related impairment in LTP [28]. It is also possible that dietary manipulation affects the activity of $\Delta 5$-desaturase that catalyzes formation of arachidonic acid from $\gamma$-linolenic acid. Activity of this enzyme is decreased with age [3,17]. This possibility is consistent with the finding that the ratio of $\gamma$-linolenic acid to arachidonic acid was increased with age and reversed by dietary manipulation.

The data indicate that there is also an age-related decrease in the concentration of docosahexanoic acid, that was partially, but not completely, reversed by dietary manipulation. This suggests that, although docosahexanoic acid is present in the diet, it is unlikely to be primarily responsible for reversing the age-related decrease in LTP, a finding that is consistent with other observations from this laboratory indicating a lack of correlation between docosahexanoic acid concentration and LTP [27]. The concentrations of a less unsaturated fatty acid, linoleic acid, as well as the saturated fatty acid, palmitic acid, were increased in hippocampal tissue prepared from aged rats compared with 
(A) $[\alpha-$ tocopherol]

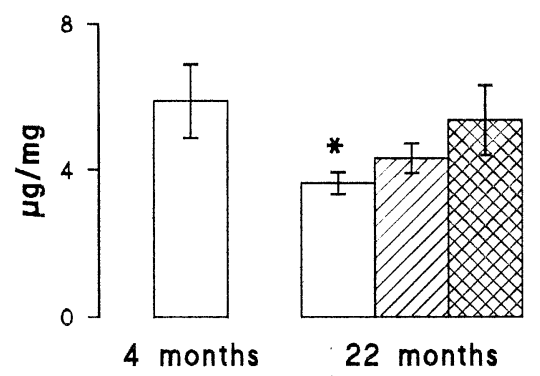

(B) $[i L-1 \beta]$

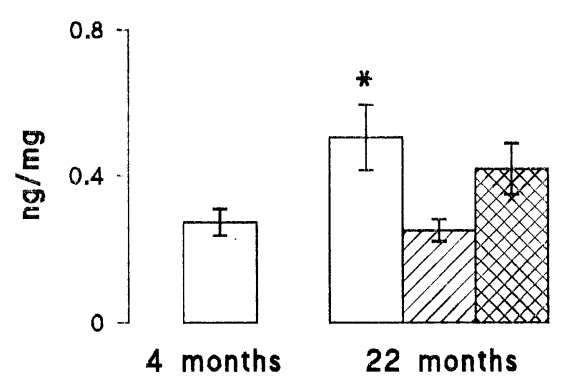

Control diet

$100 \%$ diet

$50 \%$ diet

Fig. 4. Dietary supplementation reversed age-related changes in $\alpha$-tocopherol, IL- $1 \beta$ and superoxide dismutase. The concentrations of vitamin E (a) and IL- $1 \beta$ (b) and the activity of superoxide dismutase (SOD: c) were similar in hippocampal tissue prepared from both groups of young rats and therefore the data are pooled ( $n=16$ in total). The age-related decrease in $\alpha$-tocopherol concentration and the age-related increase in IL- $1 \beta$ concentration in tissue prepared from aged rats fed on the control diet $(* p<0.05$ : Student's $t$-test for independent means; $n=11)$ were reversed in tissue prepared from aged rats fed on the experimental diets ( $n=9$ for $50 \%$ diet and $n=11$ for $100 \%$ diet). Superoxide dismutase activity was significantly increased in aged rats fed on the control $\operatorname{diet}\left({ }^{*} p<0.05\right.$ : Student's $t$-test for independent means) but dietary manipulation reversed the age-related change.

young rats. These data may indicate that during ageing there is a gradual replacement of highly unsaturated fatty acids with less unsaturated and saturated fatty acids, accounting for the increase in membrane rigidity that has been described in aged tissues [40].

$\mathrm{KCl}$-stimulated release of endogenous glutamate in synaptosomes prepared from either subgroup of young rats, was significantly greater in tetanized tissue compared with untetanized tissue, confirming previous results $[8,24]$, whereas the occlusion of the stimulatory effect arachidonic acid and ACPD enhanced KCl-stimulated release in synaptosomes prepared from tetanized dentate gyrus has also been reported [24]. Dietary manipulation in young rats did not affect these measures. KCl-stimulated release was attenuated in synaptosomes of dentate gyrus prepared from either untetanized or tetanized tissue of aged rats fed on the control diet, whereas arachidonic acid and ACPD were without effect. In marked contrast to these findings, the stimulatory effect of $\mathrm{KCl}$, and the enhancing effect of arachidonic acid and ACPD on KCl-stimulated release, were clearly evident in synaptosomes prepared from untetanized dentate gyrus of aged rats that had been fed on the experimental diets. Similarly, the increase in KCl-stimulated release and the occlusion of the effect of arachidonic acid and ACPD, were both evident in synaptosomes of tetanized dentate gyrus prepared from aged rats that received the dietary supplement. It is likely that the impaired responses are a consequence of decreased membrane fluidity, because fusion of synaptic plasma and synaptic vesicle membranes, that is a necessary step leading to release, is likely to be sensitive to fluidity changes in membrane.

Although an age-related decrease in arachidonic acid (and docosahexanoic acid) is likely to affect membrane fluidity, its putative role as retrograde messenger in LTP [6,39], suggests other actions might be predicted. It has been proposed that arachidonic acid is released from a postsynaptic site [11] into the synaptic cleft [20] and acts presynaptically to increase glutamate release $[16,23,24]$, upon activation of PLC $\gamma$ and PLC $\beta$ [26]. Thus an age-related decrease in arachidonic acid concentration is likely to limit tetanus-induced liberation of arachidonic acid from membrane phospholipids and therefore contribute to the agerelated compromise in glutamate release.

In view of the evidence suggesting that $\alpha$-lipoic acid exerts antioxidant effects [4], we considered that the beneficial effects of this diet might be a consequence of such action. We report here that there was an age-related decrease in $\alpha$-tocopherol concentration, that was completely reversed by dietary manipulation with the $50 \%$ diet, and partially reversed by the $100 \%$ diet. The mechanism underlying this effect remain to be established; possibilities are that $\alpha$-lipoic acid, acting as a free-radical scavenger [4], prevents accumulation of reactive oxygen species, but it has also been shown to regenerate $\alpha$-tocopherol [4] and to decrease lipid peroxidation [30], that might limit oxidation of $\alpha$-tocopherol.

Previous experimental data indicated that the age-related decrease in $\alpha$-tocopherol concentration was accompanied by an increase in concentration of the proinflammatory cytokine, IL-1 $\beta$ (28), whereas in vitro evidence indicated that IL- $1 \beta$ induced an increase in ROS [28,31]. The present results confirm that there was an age-related increase in IL-1 $\beta$ concentration in hippocampus [28,29]; such a change, that has the capacity to increase ROS, coupled with 
decrease in the scavenger $\alpha$-tocopherol, is consistent with reports of oxidative changes in the aged brain $[35,37,40]$. We observed that dietary manipulation reversed the agerelated increase in IL- $1 \beta$ concentration in hippocampus. We have recently found that IL-1 $\beta$ stimulates activity of superoxide dismutase in vitro and, consistent with this, is the present finding that the age-related increase in IL- $1 \beta$ concentration is accompanied by an increase in superoxide dismutase activity, whereas both effects are absent in tissue prepared from aged rats fed on the experimental diet. The action of the diet on superoxide dismutase activity identifies another potential antioxidant effect of $\alpha$-lipoic acid because an increase in activity of superoxide dismutase in the absence of parallel changes in glutathione peroxidase or catalase, might allow accumulation of hydrogen peroxide that, in the presence of metal ions, leads to formation of hydroxyl radicals.

The present findings do not identify the primary action of the $\alpha$-lipoic acid-rich diet but are consistent with the view that it exerts an antioxidant effect. The data also indicate parallel decreases in $\alpha$-tocopherol and arachidonic acid concentrations that are coupled with increased IL- $1 \beta$ concentration and increased superoxide dismutase activity in the aged brain. A causal relationship between these parameters is suggested by the diet-induced reversal of these agerelated effects.

The primary finding of this study is that supplementation with a diet enriched in $\alpha$-lipoic acid reverses several agerelated changes in synaptic function in rat hippocampus. Thus age-related impairments in LTP and glutamate release were reversed adding to the previous evidence that these measures are tightly coupled. The data are consistent with the proposal that these compromises in synaptic function might be a consequence of age-related decreases in membrane fluidity and antioxidative defenses, possibly triggered by increased IL- $1 \beta$ concentration.

\section{References}

[1] Barnes CA. Memory deficits associated with senescence: a neurophysiological and behavioral study in the rat. J Comp Physiol Psych 1979;93:74-104.

[2] Barnes CA. Effects of aging on the dynamics of information processing and synaptic weight changes in the mammalian hippocampus. Prog Brain Res 1990;86:89-104.

[3] Biagi PL, Bordoni A, Hrelia S, Celadon M, Horrobin DF. $\gamma$-Linolenic acid dietary supplementation can reverse the aging influence on rat liver microsome $\Delta^{6}$-desaturate activity. Biochim Biophys Acta 1991; 1083:187-92.

[4] Bienwenga GP, Haenen GR, Bast A. The pharmacology of the antioxidant lipoic acid. Gen Pharmacol 1997;29:315-31.

[5] Bierhaus A, Chevion S, Chevion M, Hofmann M, Quehenberger P, Illmer T, Luther T, Berentshtein E, Tritschler H, Muller M, Wahl P, Ziegler R, Nawroth PP. Advanced glycation end product-induced activation of NF-kappa B is suppressed by alpha-lipoic acid in cultured endothelial cells. Diabetes 1997;46:1482-90.

[6] Bliss TVP, Collingridge GL. A synaptic model of memory: long-term potentiation in the hippocampus. Nature 1993;361:31-9.
[7] Bradford MM. A rapid and sensitive method for the quantitation of microgram quantities of proteins utilizing the principle of protein dye binding. Anal Biochem 1976;72:248-54.

[8] Canevari L, Richter-Levin G, Bliss TVP. LTP in the dentate gyrus is associated with a persistent NMDA receptor-dependent enhancement of synaptosomal glutamate release. Brain Res 1994;667:115-7.

[9] Cao X, Phillis JW. The free radical scavenger, alpha lipoic acid, protects against cerebral ischemia-reperfusion injury in gerbils. Free Radic Res 1995;23:265-79.

[10] Choi JH, Yu BP. Brain synaptosomal aging: free radicals and membrane fluidity. Free Radic Biol Med 1995;18:133-9.

[11] Clements MP, Bliss TVP, Lynch MA. Increase in arachidonic acid in a postsynaptic membrane fraction following the induction of longterm potentiation in the dentate gyrus. Neuroscience 1991;45:37989.

[12] Davis S, Markowska AL, Wenk GL, Barnes, CA Acetyl-L-carnitine. Behavioural, electrophysiological and neurochemical effects. Neurobiol Aging 1993;14:107-5.

[13] deToledo-Morrell L, Morrell F. Electrophysiological markers of aging and memory loss in rats. Ann NY Acad Sci 1985;444:296-311.

[14] Dupree DL, Bradley J, Turner DA. Age-related alterations in potentiation in the CA1 region in F344 rats. Neurobiol Aging 1993;14: 249-58.

[15] Haan EA, Bowen DM. Protection of neocortical prisms from freezethaw injury by dimethylsulphoxide. J Neurochem 1981;37:243-6.

[16] Herrero I, Miras-Portugal MT, Sanchez-Prieto J. Positive feedback of glutamate exocytosis by metabotropic presynaptic receptor stimulation. Nature 1992;360:163-6.

[17] Horrobin DF. Loss of delta-6-desaturase activity as a key factor in aging. Med Hypotheses 1981;7:1211-20.

[18] Landfield PW, McGaugh JL, Lynch G. Impaired synaptic potentiation processes in the hippocampus of aged, memory-deficient rats. Brain Res 1978;150:85-101.

[19] Lynch MA. Age-related impairment in long-term potentiation in hippocampus: a role for the cytokine, IL-1 $\beta$ ? Prog Neurobiol 1998; 56:1-19.

[20] Lynch MA, Clements MP, Voss KL, Bramham CR, Bliss TVP. Is arachidonic acid a retrograde messenger in long-term potentiation? Biochem Soc Trans 1991;19:391-86.

[21] Lynch MA, Voss KL. Membrane arachidonic acid concentration correlates with age and induction of long-term potentiation in the dentate gyrus in the rat. Eur J Neurosci 1994;6:1008-14.

[22] Miwa H, Yamamoto M, Nishida T. Assay of free and total fatty acids (as 2-nitrophenylhydrazides) by high-performance liquid chromatography (HPLC). Clin Chim Acta 1996;155:95-102.

[23] McGahon B, Lynch M. A study of the synergism between metabotropic glutamate receptor activation and arachidonic acid in the rat hippocampus. NeuroReport 1994;5:2353-57.

[24] McGahon B, Lynch MA. The synergism between metabotropic glutamate receptor activation and arachidonic acid on glutamate release is occluded by induction of long-term potentiation in dentate gyrus. Neuroscience 1996;72:847-55.

[25] McGahon B, Clements MP, Lynch MA. The ability of aged rats to sustain long-term potentiation is restored when the age-related decrease in membrane arachidonic acid concentration is reversed. Neuroscience 1997;81:9-16.

[26] McGahon B, Lynch MA. Analysis of the interaction between arachidonic acid and metabotropic glutamate receptor activation reveals that phospholipase $\mathrm{C}$ acts as a coincidence detector in the expression of long-term potentiation in the rat dentate gyrus. Hippocampus 1998;8:1-9.

[27] McGahon BM, Martin DSD, Horrobin DF, Lynch MA. Age-related changes in synaptic function: analysis of the effect of dietary supplementation with $\omega$-3 fatty acids. Neuroscience 1999;94:305-14.

[28] Murray C, Lynch MA. Analysis of the mechanism by which dietary supplementation with vitamin $\mathrm{E}$ and vitamin $\mathrm{C}$ restores ability of aged 
animals to sustain long-term potentiation in dentate gyrus. J Biol Chem 1998a;273:12161-8.

[29] Murray C, Lynch MA. Evidence that increased hippocampal expression of the cytokine, IL- $1 \beta$, is a common trigger for age and stressinduced impairments in long-term potentiation. J Neurosci 1998b;18: 2974-81.

[30] Nickander KK, McPhee BR, Low PA, Tritschler H. Alpha-lipoic acid: antioxidant potency against lipid peroxidation of neural tissue in vitro and implications for diabetic neuropathy. Free Radic Biol Med 1996;21:631-9.

[31] O'Donnell E, Lynch MA. Analysis of age-related changes in antioxidant defense mechanisms in the rat hippocampus. Soc Neurosci Abstr 1998;24:1494.

[32] Ordronneau P, Abdullah L, Petruse P. An efficient enzyme immunoassay for glutamate using glutaraldehyde coupling of the hapten to microtiter plates. J Immunol Meth 1991;142:169-76.

[33] Packer L. Alpha-lipoic acid: a metabolic antioxidant which regulates NF-kappa B signal transduction and protects against oxidative injury. Drug Metab Rev 1998;30:245-75.
[34] Panigrahi M, Sadguna Y, Shivakumar BR, Kolluri SV, Roy S, Packer L, Ravindranath V. Alpha-lipoic acid protects against reperfusion injury following cerebral ischemia in rats. Brain Res 1996;717: $184-8$.

[35] Reiter RJ. Oxidative processes and antioxidative defense mechanisms in the aging brain. FASEB J 1995;9:526-533.

[36] Spitz D R, Oberley LW. An assay for superoxide dismutase activity in mammalian tissue homogenates. Anal Biochem 1989;179:8-18.

[37] Urano S, Asai Y, Makabe S, Matsuo M, Izumiyama N, Ohtsubo K, Endo T. Oxidative injury of synapse and alteration of antioxidative defense systems in rats, and its prevention by vitamin E. Eur J Biochem 1997;245:64-70.

[38] Vatassery GT. In vitro oxidation of vitamins $\mathrm{C}$ and $\mathrm{E}$, cholesterol and thiols in rat brain synaptosomes. Lipids 1994;30:1007-13.

[39] Williams JH, Errington ML, Lynch MA, Bliss TVP. Arachidonic acid induces a long-term activity dependent enhancement of synaptic transmission in the hippocampus. Nature 1989;341:739-42.

[40] Zs.-Nagy I. The membrane hypothesis of aging. London: CRC Press Inc., 1994. 